\title{
ON UNITARY REPRESENTABILITY OF TOPOLOGICAL GROUPS
}

\author{
JORGE GALINDO
}

\begin{abstract}
We prove that the additive group $\left(E^{*}, \tau_{k}(E)\right)$ of an $\mathcal{L}_{\infty}$-Banach space $E$, with the topology $\tau_{k}(E)$ of uniform convergence on compact subsets of $E$, is topologically isomorphic to a subgroup of the unitary group of some Hilbert space (is unitarily representable). This is the same as proving that the topological group $\left(E^{*}, \tau_{k}(E)\right)$ is uniformly homeomorphic to a subset of $\ell_{2}^{\kappa}$ for some $\kappa$.

As an immediate consequence, preduals of commutative von Neumann algebras or duals of commutative $C^{*}$-algebras are unitarily representable in the topology of uniform convergence on compact subsets. The unitary representability of free locally convex spaces (and thus of free Abelian topological groups) on compact spaces, follows as well.

The above facts cannot be extended to noncommutative von Neumann algebras or general Schwartz spaces.
\end{abstract}

\section{INTRODUCTION}

We address in this paper the problem of determining which topological groups embed in the unitary group of a Hilbert space. This problem is relevant in the program of extending Harmonic Analysis beyond locally compact groups (see [23] or [24], see also [28]), and strongly related to other much studied problems like the uniform embeddability of metric or Banach spaces in $\ell_{2}$, see [6, Chapter 7], or the universality of orbit equivalence relations induced by actions of the unitary group, see $[14,15]$.

When a topological group can be embedded in the unitary group of a Hilbert space (unitary groups will be assumed to carry the strong operator topology) we say that it is unitarily representable. Positive definite functions play a prominent rôle in the unitary representability of a topological group. This is mainly because the (strong operator) topology of the unitary group is determined by positive definite functions. As a matter of fact, a topological group is unitarily representable precisely when its topology is generated by its continuous positive definite functions as happens, for instance, with discrete groups (characteristic functions of points are linear combinations of positive definite functions), see Lemma 2.1. All locally compact groups are actually unitarily representable, their regular representation $\lambda$ establishes a topological isomorphism $\lambda: G \rightarrow \mathcal{U}\left(L_{2}(G)\right)$. If we go beyond the

2000 Mathematics Subject Classification. 43A35,46B99, 22A10, 54H11, 54E35.

Key words and phrases. Unitary group, positive definite, $\mathcal{L}_{\infty}$-Banach space, free Abelian topological group, free locally convex space, free Banach space, unitarily representable, uniform embedding, Schwartz space.

Research partially supported by Spanish Ministry of Science, grant MTM2008-04599/MTM. The foundations of this paper were laid during the authors stay at the University of Ottawa supported by a Generalitat Valenciana grant CTESPP/2004/086. 
class of locally compact groups, regular representations are no longer at reach. Megrelishvili [20] has proven that isometry groups of separable Hilbert spaces embed uniformly in $\ell_{2}$ and, therefore, that for separable Banach spaces (or even Polish groups), unitary representability implies uniform embeddability in $\ell_{2}$. This shows how strongly the problem of unitary representability is linked with another problem that has been the object of deep research by many authors: the uniform classification of Banach spaces. Both problems are indeed equivalent for Abelian groups, see Theorem 2.3. For non-Abelian groups it remains unknown whether these two problems remain equivalent.

We will consider here the unitary representability of additive groups of Banach spaces equipped with topologies of uniform convergence on compact sets, the natural topology in the duality theory of topological groups. Denote by $\tau_{k}(E)$ the topology of uniform convergence on compact subsets of a topological space $E$. We prove in Section 3 that for an arbitrary Banach $\mathcal{L}_{\infty}$-space $E$, the additive group of $\left(E^{*}, \tau_{k}(E)\right)$ embeds isomorphically in a power of $\ell^{1}$ and is therefore unitarily representable. The results stated in the second paragraph of the abstract then follow. Since $\left(E^{*}, \tau_{k}(E)\right)$ is always a Schwartz locally convex space (i.e. a locally convex space that can be obtained as projective limit of Banach spaces with compact linking maps) and so are other classes of unitarily representable groups, such as nuclear spaces it is natural to study whether Schwartz spaces must be unitarily representable. We analyze this question in Section 4 and focus on Schwartz topologies on the Banach space $c_{0}$. We find that pre-images of neighbourhoods of 1 in $\mathbb{C}$ under continuous positive definite function on $c_{0}$ are always quite large. As a consequence, we obtain that positive-definite functions do not define either the norm topology or the topology of uniform convergence on compact subsets of $\ell_{1}$. Since the latter is a Schwartz topology, we conclude that Schwartz spaces need not be unitarily representable. This example also shows that dual groups of Banach spaces may fail to be unitarily representable.

We finish this introduction with some words on notation and terminology.

Unless specifically stated all topological groups will be Abelian. Given a finite measure $\mu$, the topological vector space of all complex-valued measurable functions will be denoted by $L_{0}(\mu), L_{0}(\mu, \mathbb{T})$ will stand for the subset of $L_{0}(\mu)$ consisting of $\mathbb{T}$-valued functions. Under multiplication, $L_{0}(\mu, \mathbb{T})$ becomes a topological group. We will always assume that $L_{0}(\mu)$ carries the topology of convergence in measure. Sometimes we will find useful to specify the space $X$ on which $\mu$ is defined and use $L_{0}(X, \mu)$ instead of $L_{0}(\mu)$.

By an uniform embedding between topological groups $T: G \rightarrow H$ we mean an injective mapping such that both $T$ and $T^{-1}$ are uniformly continuous.

By $\tau_{k}(X)$ we will denote the topology of uniform convergence on the compact subsets of a topological space $X$. If $E$ is a Banach space, the term dual group of $E$, in symbols $\widehat{E}$, will refer to the additive group of $\left(E^{*}, \tau_{k}(E)\right)$. In the duality theory of topological groups, the symbol $\widehat{E}$ is usually reserved for the character group of $E$, i. e. the group of continuous homomorphisms into the unit circle $\mathbb{T}$ with the topology of uniform convergence on the compact subsets of $E$. It is easy to see that this group is topologically isomorphic to $\left(E^{*}, \tau_{k}(E)\right)$.

The ball of radius $\delta$ centered in the identity of a metrizable group $G$ will be denoted by $B_{G, \delta}$, in case $\delta=1$, we will simply write $B_{G}$. 


\section{ACKNOWLEDGEMENTS}

Concerning this paper, I owe my thanks to several people that have contributed to it in different senses.

The origin of this paper is in the author's discussions with V. Pestov at the University of Ottawa in the Spring of 2004. The author is indebted to V. Pestov for proposing him the present problem and for providing the first arguments in favour of the unitary representability of $A([0,1])$.

Some observations of V. Tarieladze were very useful. M. Megrelishvili has contributed by carefully reading several drafts of this paper and pointing out some inaccuracies. W. B. Johnson suggested the final form of Theorem 3.2 and therefore the final tenor of the paper.

I am finally indebted to V. Uspenskiufor finding a wrong statement in a previous version of this paper and for sharing with me an elegant, direct proof of the unitary representability of $L(X)$ for completely regular $X$.

\section{UNITARY REPRESENTABILITY OF TOPOLOGICAL GROUPS}

We collect here some facts available in the literature on unitary representability of topological groups.

Positive-definite functions provide an internal criterion for the unitary representability of a topological group, see Lemma 2.1, and therefore constitute an essential tool. A complex-valued function $\phi$ defined on a topological group $G$ is positive definite provided that for every finite subset $\left\{x_{j}\right\}_{j=1}^{n}$ of $G$ and every collection of complex numbers $\left\{\lambda_{j}\right\}_{j=1}^{n}$,

$$
\sum_{i, j=1}^{n} \lambda_{i} \bar{\lambda}_{j} \phi\left(x_{i} x_{j}^{-1}\right) \geq 0
$$

The function $e^{-\|\cdot\|}$ defined on a Hilbert space, is a positive definite function of special importance. Its positive definiteness is usually proven as a consequence of Schoenberg's theorem on exponentiation of so-called negative-definite functions, see [7, Chapter 3]. This function suffices to note one direction of the following well-known characterization of unitary representability through positive definite functions.

Lemma 2.1. A topological group $G$ is unitarily representable if and only if for each neighbourhood $U$ of the identity 0 of $G$, there is a real-valued positive definite function $\phi_{U}$ with $\phi_{U}(0)=1$ such that

$$
\left\{g \in G:\left|\phi_{U}(g)-1\right|<1 / 2\right\} \subseteq U .
$$

Proof. Sufficiency of the condition is an easy consequence (see Theorem 2.1 of [14]) of the GNS construction that associates a unitary representation to each positivedefinite function, see for instance [5, Appendix C] or [12, Theorem 3.20].

Assume now that $G$ is unitarily representable and let $T: G \rightarrow \mathcal{U}(\mathbb{H})$ denote a topological isomorphism of $G$ into the unitary group of some Hilbert space $\mathbb{H}$. Consider $\widetilde{U}$ a neighbourhood of the identity in $\mathcal{U}(\mathbb{H})$ such that $T(U)=\widetilde{U} \cap T(G)$. Since the topology of $\mathcal{U}(\mathbb{H})$ is the strong operator topology, there will be some 
$\varepsilon_{i}>0$ and $\xi_{i} \in \mathbb{H}, 1 \leq i \leq n$, such that

$$
\widetilde{U} \supset \bigcap_{i=1}^{n}\left\{S \in \mathcal{U}(\mathbb{H}):\left\|S \xi_{i}-\xi_{i}\right\| \leq \varepsilon_{i}\right\} .
$$

Now define $\phi_{U}=e^{-\log 2 \sum_{i=1}^{n} \frac{1}{\varepsilon_{i}}\left\|T(\cdot) \xi_{i}-\xi_{i}\right\|}$.

Schoenberg [27] not only realized that $e^{-\|\cdot\|}$ is a positive definite function on any $L_{2}(\mu)$, he also discovered that raising the norm to a power $\alpha<1$ did not change the positive definiteness of the norm and completely classified $L_{p}$-spaces in terms of the positive definiteness of $e^{-\|\cdot\|}$.

This classification is contained in the following theorem that summarizes several known results about unitary representability of Banach spaces.

Theorem 2.2 (Aharoni, Maurey and Mityagin [1], Megrelishvili [20], Schoenberg [27], see Chapter 8 of [6]). If a Banach space is unitarily representable, then it can be isomorphically embedded in $L_{0}(\mu)$ for some measure $\mu$ and must have cotype 2. An infinite-dimensional Banach space $L_{p}(\mu)$ is unitarily representable if and only if $1 \leq p \leq 2$.

Proof. (Sketch) Megrelishvili [20] proves that $U\left(\ell_{2}\right)$ embeds uniformly in $\ell_{2}$ and Aharoni, Maurey and Mityagin prove in [1] that a Banach space admits a uniform embedding in $\ell_{2}$ if and only if it admits an isomorphic embedding in $L_{0}(\mu)$. Since (1) $e^{-\|\cdot\|}$ is positive definite on $L_{p}$ for $1 \leq p \leq 2$ (Schoenberg), (2) every Banach space isomorphic to a subspace of $L_{0}(\mu)$ has cotype 2 ([6, Corollary 8.17]) and (3) infinite dimensional $L_{p}(\mu)$-spaces with $p>2$ do not have cotype 2 , all the assertions in the theorem follow.

Gathering the information of these two Sections, we obtain the following characterization of unitary representability.

Theorem 2.3. Let $G$ be an Abelian topological group. The following assertions are equivalent:

(1) $G$ is unitarily representable.

(2) $G$ is topologically isomorphic to a subgroup of $L_{0}(X, \mu, \mathbb{T})$, for some compact $X$ and some Borel measure $\mu$ on $X$.

(3) There is a uniform embedding of $G$ into a power $\left(\ell_{1}\right)^{\kappa}$ of the Banach space $\ell_{1}$.

(4) There is a uniform embedding of $G$ into a power $\left(\ell_{2}\right)^{\kappa}$ of the Banach space $\ell_{2}$.

Proof. The equivalence between (1) and (2) is well-known and appears for instance in [15] for Polish groups. For non-Polish groups the argument is still valid.

Megrelishvili [20] proves that the unitary group $\mathcal{U}\left(\ell_{2}\right)$ embeds uniformly in $\ell_{2}$. Since the unitary group $\mathcal{U}\left(\ell_{2}(\kappa)\right)$ of a $\kappa$-dimensional Hilbert space is topologically isomorphic to a subgroup of the product $\prod_{\kappa} \mathcal{U}\left(\ell_{2}\right)$, this shows that (1) implies (4).

That (1) follows from (4) is a pretty straightforward consequence of the results of [1], and might have been known to some specialists. We sketch the proof of this fact.

Assume that (4) holds. Let $\ell_{2, i}$ be a copy of $\ell_{2}$ for every $i<\kappa$ and consider a uniform embedding $T: G \rightarrow \prod_{i<\kappa} \ell_{2, i}$. Let also $\left\{A_{j}: j<\kappa\right\}$ be an enumeration 
of all finite subsets of $\kappa$ and define the corresponding projections $\pi_{j}: \prod_{i<\kappa} \ell_{2, i} \rightarrow$ $\prod_{i \in A_{j}} \ell_{2, i}$. The norm of $\prod_{i \in A_{j}} \ell_{2, i}\left(\cong \ell_{2}\right)$ will be denoted by $\|\cdot\|_{j}$.

We now adapt Corollary 3.6 of [1] (see also Chapter 8 of [6]).

Let $M$ denote an invariant mean on $G$ (i.e. a continuous functional on $\ell^{\infty}(G)$ with $M(1)=1$ and $M\left(L_{x} f\right)=M(f)$, if $L_{x} f(y)=f(x y)$ ). Each projection $\pi_{j}$ defines a continuous positive definite function $\phi_{j}$ given by

$$
\phi_{j}(g)=M\left(f_{g}\right), \quad \text { with } \quad f_{g}(y)=e^{-\left\|\pi_{j} T(g y)-\pi_{j} T(y)\right\|_{j}^{2}} .
$$

To avoid confusions, we have adopted here multiplicative notation for the group operation on $G$ and additive notation for $\ell^{2}$. That $\phi_{j}$ is positive definite follows from the invariance of $M$ and that $\phi_{j}$ is continuous follows from the uniform continuity of $\pi_{j} T$, see Corollary 3.6 of [1]. Since $T^{-1}$ is uniformly continuous as well, every neighbourhood $U$ of the identity in $G$ determines a projection $\pi_{j}$ and an $\varepsilon>0$ such that

$$
\left\{g:\left\|\pi_{j} T(g)-\pi_{j}(\bar{a})\right\|_{j}<\varepsilon\right\} \subset\left\{g: g \in T^{-1}(\bar{a}) U\right\} \quad \text { for every } \bar{a} \in T(G) \subset\left(\ell_{2}\right)^{\kappa} .
$$

Arguing again as in Corollary 3.6 of [1] it follows that there is $\delta>0$ such that

$$
\left\{g \in G:\left|\phi_{i}(g)-1\right|<\delta\right\} \subset U .
$$

Hence, $G$ is unitarily representable by Lemma 2.1 .

That assertions (4) and (3) are equivalent is indeed a well-known fact. That (3) implies (4) is proved in [1], since $\ell_{2}$ embeds even isometrically in $\ell_{1},(4)$ implies (3).

Corollary 2.4. An Abelian Polish group is unitarily representable if and only if it is uniformly embeddable in $\ell_{2}$.

Remark 2.5. As M. Megrelishvili [19] has indicated to the author, Corollary 2.4 is true for Polish amenable groups, this readily follows from the proof of the implication (4) $\Longrightarrow(1)$ of Theorem 2.3. According to this same letter, Megrelishvili announced that (4) implies (1) in 2002.

Corollary 2.6. The additive group of a Banach space is unitarily representable if and only if it is both linearly isomorphic to a subspace of $L_{0}(\mu)$ and topologically isomorphic to a subgroup of $L_{0}(\mu, \mathbb{T})$.

\section{Dual groups of $\mathcal{L}_{\infty}$-SPACeS}

We show in this section that the dual space $E^{*}$ of an $\mathcal{L}_{\infty}$-space $E$ with the topology of uniform convergence on compact subsets of $E, \tau_{k}(E)$, is always unitarily representable.

Recall that $\mathcal{L}_{\infty}$-spaces are Banach spaces whose finite dimensional subspaces are close to $\ell_{\infty}^{m}$-spaces, see for instance [6, Appendix F] for the actual definition. For our purposes it suffices to say that $C(K)$-spaces are $\mathcal{L}_{\infty}$ spaces for every compact space $K$.

Lemma 3.1 (Theorem 4.3 and Remark 4.5 of [13]). Let $E$ and $Y$ be $\mathcal{L}_{\infty}$-spaces, $Y$ separable. For each compact subset $K$ of $E$ there is a one-to-one compact operator $T: Y \rightarrow E$ with $K \subset T\left(B_{Y}\right)$.

Corollary 3.2. Let $E$ be a $\mathcal{L}_{\infty}$ space and let $K \subset E$ be compact. There is then a compact one-to-one operator $T_{K}: c_{0} \rightarrow E$ with $K \subset T_{K}\left(B_{c_{0}}\right)$. 
Theorem 3.3. The dual group $\widehat{E}=\left(E^{*}, \tau_{k}(E)\right)$ of a $\mathcal{L}_{\infty}$-space $E$ embeds in a power of $\ell^{1}$, and hence is unitarily representable.

Proof. Let $\mathcal{K}(E)$ denote a set that is cofinal in the family of all compact subsets of $E$ (ordered by inclusion). For each $K \in \mathcal{K}(E)$ we consider the compact operator $T_{K}: c_{0, K} \rightarrow E$ defined on a copy $c_{0, K}$ of $c_{0}$ that is provided by Corollary 3.2.

Define now

$$
\Psi: E^{*} \rightarrow \prod_{K \in \mathcal{K}(E)} \ell_{1, K}
$$

as the product $\Psi=\prod_{K \in \mathcal{K}(E)} T_{K}^{*}$, where again $\ell_{1, K}$ represents a copy of $\ell_{1}$. We now check that $\Psi$ is one-to-one, $\tau_{k}(E)$-continuous and open.

Suppose $\Psi(f)=0$, and take any $x \in E$. By considering some $K \in \mathcal{K}(E)$ with $x \in K$, we see that $f(x)=0$, and therefore that $\Psi$ is injective.

To see that $\Psi$ is $\tau_{k}$-continuous, we must check that the conjugate operators $T_{K}^{*}$ are all $\tau_{k}(\mathrm{E})$-continuous. Taking into account that $T_{K}$ is a compact operator, we have that $T_{k}\left(B_{c_{0, K}}\right) \subset K_{0}$ for some $K_{0} \in \mathcal{K}(E)$. Uniform convergence on $K_{0}$ will therefore imply uniform convergence on $B_{c_{0, K}}$ (and thus norm-convergence on $\left.\ell_{1, K}\right)$. Accordingly $T_{K}^{*}$ is $\tau_{k}$-continuous.

As to the openness of $\Psi$, it is sufficient to observe that uniform convergence on every $B_{c_{0, K}}$ implies $\tau_{k}(E)$-convergence, since every $K \in \mathcal{K}(E)$ is contained in the $T_{K}$-image of the corresponding $B_{c_{0, K}}$.

Corollary 3.4. If a topological group $G$ admits a uniform embedding into $\left(L_{1}(X, \mu), \tau_{k}(C(X))\right)$ for some compact $X$ and some Borel measure $\mu$ on $X$, then $G$ is unitarily representable

Corollary 3.5. Let $A$ denote a commutative Banach algebra, and let $\mathcal{M}$ denote a commutative von Neumann algebra with predual $\mathcal{M}_{*}$. The additive groups of $A^{*}$ and $\mathcal{M}_{*}$ are both unitarily representable for the respective topologies of uniform convergence on compact subsets $\tau_{k}(A)$ and $\tau_{k}(\mathcal{M})$.

Theorem 3.3 can be directly applicable to free locally convex spaces and free Abelian topological groups on compact spaces. If $X$ is a completely regular space, the free locally convex space $L(X)$ and the free Abelian topological group $A(X)$ on $X$ are obtained by providing the free vector space (resp. the free Abelian group on $X)$ with a locally convex vector space topology (resp. a topological group topology) such that every continuous function $f: X \rightarrow E$ into a locally convex space (resp. a topological grouop) can be extended to a continuous linear map $\bar{f}: L(X) \rightarrow E$ (resp. to a continuous homomorphism), this is thus a linearization process. What is important for our purposes is that these free topological objects can be realized as preduals of $C(X, \mathbb{R})$ and $C(X, \mathbb{T})$ respectively: $C(X, \mathbb{R})$ appears as the space of continuous linear functionals on $L(X)$ and $C(X, \mathbb{T})$ appears as the space of continuous characters on $A(X) . L(X)$ carries the topology of uniform convergence on equicontinuous pointwise bounded subsets of, $C(X, \mathbb{R})$ and $A(X)$ the topology of uniform convergence on equicontinuous subsets of $C(X, \mathbb{T})$, see [31], and [22].

The dual group of $C(X, \mathbb{R})$ will be denoted as $M_{c}(X)$. Since the topology of $L(X)$ is the topology of uniform convergence on equicontinuous pointwise bounded (=relatively compact) sets, $L(X)$ is a subgroup of $M_{c}(X)$ in a natural way. By Tkachenko-Uspenskiil's theorem [29,30], $A(X)$ is a topological subgroup of $L(X)$ and thus of $M_{c}(X)$. The following is then an immediate corollary to Theorem 3.3, it answers Question 35 in [25], see also Question 6.10 in [21] 
Corollary 3.6. The additive group of $M_{c}(K)$ and its subgroups $L(K)$ and $A(K)$ are unitarily representable for every compact Hausdorff space $K$.

Remark 3.7. In response to a previous version of this paper, Uspenskiı[[32] has provided a different argument for the unitary representabilty of $L(X)$, valid for any completely regular space $X$. The preprint is now available [33].

Remark 3.8. For a given metric space $(K, d)$, the free locally convex space over $K$ can be obtained as the projective limit of the free Banach spaces over $\left(K, d_{\alpha}\right)$ for some family of distances $d_{\alpha}$ compatible with $d$ (see e.g. [15] for the precise meaning of the term free Banach). For that reason, it was expected that Corollary 3.6 should follow from an analogous statement for some class of free Banach spaces $F B\left(K, d_{f}\right)$, but, somewhat unexpectedly, the proof of Theorem 3.3 does not allow such a deduction. Examples of free Banach spaces that are not unitarily representable are actually easy to come by. For instance every Banach space $X$ embeds uniformly in $F B(X)$ (there is even a linear isometry of $X$ into $\mathrm{FB}(\mathrm{X})$ when $X$ is separable, see [16]), and thus free Banach spaces on nonunitarily representable Banach spaces are not unitarily representable themselves. The compact space defined in [10] shows that free Banach spaces on compact metric spaces need not be unitarily representable either. It is on the other hand unclear what happens with $F N\left(\mathbb{I}, d_{f}\right)$ for arbitrary metric transforms $d_{f}$ (i.e. metrics that arise after composing $d$ with an increasing, concave function $f: \mathbb{I} \rightarrow \mathbb{I}$ ) and therefore whether unitary representability of compact spaces can be deduced from unitary representability of free Banach spaces. While it is known that, for $f_{\alpha}(t)=t^{\alpha}, F B\left(\mathbb{I}, d_{f_{\alpha}}\right)$ is isomorphic to $\ell_{1}$ and hence unitarily representable ([8] or [34], see also the recent complete account of [17]), the available proofs of this fact cannot be extended to all metric transforms.

\section{ON THE LIMITS OF THE CLASS OF UNITARILY REPRESENTABLE GROUPS}

We try here to get an idea of which are the limits of the class of unitarily representable groups. We have shown in Section 2 that dual groups of Banach $\mathcal{L}_{\infty^{-}}$ spaces are in that class. Another distinguished family of unitarily representable groups is the variety of nuclear groups that contains additive groups of nuclear locally convex spaces and locally compact Abelian groups, see [3]. The unitary representability of nuclear groups was obtained in [4]; in the particular case of nuclear locally convex spaces, this follows directly from their representation as projective limits of Hilbert spaces.

It is not clear how to extend the class of unitarily representable groups beyond nuclear groups or dual groups of Banach $\mathcal{L}_{\infty}$-spaces, as neighbouring families already contain groups that are not unitarily representable.

One possible extension could involve a noncommutative version of Corollary 3.5. But Corollary 3.5 is based on embedding the dual group $\left(A^{*}, \tau_{k}(A)\right)$ of a commutative von Neumann algebra $A$ in a product of preduals of commutative von Neumann algebras (namely $\ell_{1}$ 's). This point of view cannot be carried over to the noncommutative case, as already preduals of noncommutative von Neumann algebras may fail to be unitarily representable. The algebra of trace-class operators $C_{1}$, which is the predual of the von Neumann algebra $B\left(\ell_{2}\right)$ of all bounded operators on $\ell_{2}$, is one example, see the Remark in page 194 of [6]. 
Another possible extension could involve Schwartz spaces. Dual groups of $\mathcal{L}_{\infty^{-}}$ spaces and nuclear spaces both belong to the class of Schwartz spaces (or to its topological group-theoretic analog Schwartz groups [2] for the case of nuclear groups). Recall that a locally convex space is a Schwartz space if it can be represented as a projective limit of Banach spaces with compact linking maps. Notwithstanding its closeness to theses classes, the class of Schwartz spaces, also contains groups that are not unitarily representable. Our example will be the additive group of $\left(c_{0}, \tau_{k}\left(\ell_{1}\right)\right)$. This is in fact a universal generator for the class of Schwartz spaces. Since compact subsets of $\ell_{1}$ are contained in the closed convex hull of null sequences, $\tau_{k}\left(\ell_{1}\right)$ can be replaced by the topology of uniform convergence on null-sequences in $\ell^{1}$. We denote by $\mathcal{S}\left(c_{0}\right)$ the vector space $c_{0}$ equipped with this topology.

Neighbourhoods of 0 in $\mathcal{S}\left(c_{0}\right)$, are determined by sequences of numbers going to 0 : given such a sequence $\bar{\alpha}=\left(\alpha_{n}\right)_{n}$, we consider the corresponding neighbourhood

$$
U_{\bar{\alpha}}=\left\{\left(x_{n}\right)_{n} \in c_{0}:\left|x_{n}\right| \leq \frac{1}{\left|\alpha_{n}\right|}, \text { for every } n\right\} .
$$

The sets $U_{\bar{\alpha}}$ (with $\alpha \in c_{0}$ ) constitute a neighbourhood basis at 0 of $\mathcal{S}\left(c_{0}\right)$, see [26].

To see why $\mathcal{S}\left(c_{0}\right)$ is not unitarily representable we need a couple of results on the structure of linear operators with values in $L_{0}$.

Theorem 4.1 (Nikishin factorization theorem, see for instance Theorem 13 of [9] or Proposition 8.16 of [1]). Let $X$ be a Banach space. Every continuous linear operator $T: X \rightarrow L_{0}$ factorizes through $L_{q}$ for each $0<q<1$, i.e. there are continuous linear operators $S_{1}: X \rightarrow L_{q}$ and $S_{2}: L_{q} \rightarrow L_{0}$ such that $S_{2} S_{1}=T$.

In the sequel we will make use of the standard unit vectors $e_{n} \in c_{0}$. The symbol $e_{n}$ will denote, as usual, the sequence with one in the $n$th place and zero otherwise.

Theorem 4.2 (Theorem 4.3 of [18]). For every bounded operator $T: c_{0} \rightarrow L_{p}$, $0<p \leq 2, \sum\left\|T\left(e_{n}\right)\right\|^{2}<\infty$.

Theorem 4.3. The Schwartz space $\mathcal{S}\left(c_{0}\right)$ is not unitarily representable.

Proof. Let $\bar{\alpha}=\left(\alpha_{n}\right)_{n}$ denote the sequence with $\alpha_{n}=1 / \log (n+1)$ and consider the $\mathcal{S}\left(c_{0}\right)$-neighbourhood of the identity

$$
U_{\bar{\alpha}}=\left\{\left(a_{n}\right)_{n} \in c_{0}: a_{n} \leq \log (n+1), \text { for every } n\right\} .
$$

We will next see that there is no positive definite function $\phi$ on $c_{0}$ such that $\left\{\left(a_{n}\right)_{n} \in\right.$ $\left.c_{0}:\left|\phi\left(\left(a_{n}\right)_{n}\right)-1\right|<1 / 2\right\}$ is contained in $U_{\bar{\alpha}}$. By Lemma 2.1 this will prove the theorem.

By Lemma 4.2 of [1] each positive definite mapping $\phi$ on a linear topological space $X$ induces a continuous linear operator $T: X \rightarrow L_{0}$ in such a way that for every $x \in X$, the characteristic function $\varphi_{T(x)}$ of $T(x)$ satisfies $\varphi_{T(x)}(t)=\phi(t x)$ for every $t \in(0,1)$. Here we have adopted the usual probabilistic terminology and by the characteristic function of $f \in L_{0}(\mu)$ we mean the complex-valued function $\varphi_{f}(t)=\int e^{i t f(x)} d \mu(x)$. It is then easy to see that there is some $\delta>0$ such that

$$
T^{-1}\left(B_{L_{0}, \delta}\right) \subset\left\{\left(a_{n}\right)_{n} \in c_{0}:\left|\phi\left(\left(a_{n}\right)_{n}\right)-1\right|<1 / 2\right\} .
$$

Let $T: c_{0} \rightarrow L_{0}$ and $\delta>0$ be a continuous linear operator and a real number respectively. In view of (1), it will suffice to see that $T^{-1}\left(B_{L_{0}, \delta}\right) \nsubseteq U_{\bar{\alpha}}$.

Let $c_{0} \stackrel{S_{1}}{\rightarrow} L_{r} \stackrel{S_{2}}{\rightarrow} L_{0}$ be a factorization of the operator $T$ as in Theorem 4.1. By Theorem $4.2, \sum_{n}\left\|S_{1}\left(e_{n}\right)\right\|_{r}^{2}<\infty$. 
Let $\varepsilon>0$ be such that $S_{2}\left(B_{L_{r}, \varepsilon}\right) \subset B_{L_{0}, \delta}$ and choose $k$ large enough so that $\left\|S_{1}\left(e_{k}\right)\right\|_{r}<\frac{\varepsilon}{2 \log (k+1)}$. Take now some $\bar{x}=\left(x_{n}\right)_{n} \in c_{0}$ with $x_{k}=0$ and $S_{1}(\bar{x}) \leq \varepsilon / 2$ and define a sequence $\bar{a} \in c_{0}$ as $\bar{a}=\bar{x}+a_{k} e_{k}$ with $a_{k}=\frac{\varepsilon}{2\left\|S_{1}\left(e_{k}\right)\right\|_{r}}$. Clearly $\bar{a} \notin U_{\bar{\alpha}}$ and $\left\|S_{1}(\bar{a})\right\|_{r} \leq\left\|S_{1}(\bar{x})\right\|_{r}+\left|a_{k}\right|\left\|S_{1}\left(e_{k}\right)\right\|_{r} \leq \varepsilon$. We have thus that $T\left(\left(a_{n}\right)_{n}\right)=$ $S_{2} S_{1}\left(\left(a_{n}\right)_{n}\right) \in B_{L_{0}, \delta}$ while $\left(a_{n}\right)_{n} \notin U_{\bar{\alpha}}$.

It immediately follows that dual groups of Banach spaces need not be unitarily representable (and hence that Theorem 2.3 cannot be thus generalized).

Corollary 4.4. The dual group of $\ell_{1}$, i.e. $\left(\ell_{\infty}, \tau_{k}\left(\ell_{1}\right)\right)$, is not unitarily representable.

Proof. Just observe that $\mathcal{S}\left(c_{0}\right)$ embeds linearly as topological subspace of $\widehat{\ell}_{1}=$ $\left(\ell_{\infty}, \tau_{k}\left(\ell_{1}\right)\right)$.

Remark 4.5. Theorem 4.3 shows that neighbourhoods of the identity for the topology generated by continuous positive definite functions on $c_{0}$ are quite large, and hence that this topology is rather weak. The proof of Theorem 4.3 shows indeed that every continuous positive definite mapping $\phi$ on $c_{0}$ has associated a sequence $\left(b_{n}\right) \in \ell_{2}$ with $b_{n} \geq 0$ for all $n$, and some $\delta>0$, such that

$$
\left\{\left(a_{n}\right) \in c_{0}: \sum\left|a_{n}\right| b_{n}<\delta\right\} \subset\left\{\left(a_{n}\right) \in c_{0}:\left|\phi\left(\left(a_{n}\right)\right)-\phi(0)\right|<1\right\} .
$$

If $\bar{\lambda} \in c_{0} \backslash \ell_{2}$, the set $\left\{\left(a_{n}\right) \in c_{0}: \sum\left|a_{n}\right| b_{n}<\delta\right\}$ is never contained in $U_{\bar{\lambda}}$. This same argument also shows that $c_{0}$ (with its norm topology) does not embed uniformly in $\ell_{2}$, a fact that was first proved by Enflo [11].

Most of the techniques of this paper are commutative in nature, it seems nevertheless worth to end this paper with a mention to two natural questions that are left untouched here.

Question 1 (Question 4.4 of [20]). If a (non-Abelian) Polish topological group embeds uniformly in $\ell_{2}$, must it be unitarily representable?

Question 2 (Question 35 of [25]). Is the free topological group on a compact space (or even $[0,1]$ ) unitarily representable?

\section{REFERENCES}

[1] I. Aharoni, B. Maurey, and B. S. Mityagin. Uniform embeddings of metric spaces and of Banach spaces into Hilbert spaces. Israel J. Math., 52(3):251-265, 1985.

[2] L. Aussenhofer, M. J. Chasco, X. Domínguez, and V. Tarieladze. On Schwartz groups. Studia Math., 181(3):199-210, 2007.

[3] Wojciech Banaszczyk. Additive subgroups of topological vector spaces. Springer-Verlag, Berlin, 1991.

[4] Wojciech Banaszczyk. Theorems of Bochner and Lévy for nuclear groups. In Nuclear groups and Lie groups (Madrid, 1999), volume 24 of Res. Exp. Math., pages 31-44. Heldermann, Lemgo, 2001.

[5] Bachir Bekka, Pierre de la Harpe, and Alain Valette. Kazhdan's property (T), volume 11 of New Mathematical Monographs. Cambridge University Press, Cambridge, 2008.

[6] Yoav Benyamini and Joram Lindenstrauss. Geometric nonlinear functional analysis. Vol. 1. American Mathematical Society, Providence, RI, 2000.

[7] Christian Berg, Jens Peter Reus Christensen, and Paul Ressel. Harmonic analysis on semigroups. Springer-Verlag, New York, 1984. Theory of positive definite and related functions.

[8] Z. Ciesielski. On the isomorphisms of the spaces $H_{\alpha}$ and m. Bull. Acad. Polon. Sci. Sér. Sci. Math. Astronom. Phys., 8:217-222, 1960. 
[9] S. J. Dilworth. Special Banach lattices and their applications. In Handbook of the geometry of Banach spaces, Vol. I, pages 497-532. North-Holland, Amsterdam, 2001.

[10] Y. Dutrieux and G. Lancien. Isometric embeddings of compact spaces into Banach spaces. J. Funct. Anal., 255(2):494-501, 2008.

[11] Per Enflo. On a problem of Smirnov. Ark. Mat., 8:107-109, 1969.

[12] Gerald B. Folland. A course in abstract harmonic analysis. CRC Press, Boca Raton, FL, 1995.

[13] V. P. Fonf, W. B. Johnson, A. M. Plichko, and V. V. Shevchyk. Covering a compact set in a Banach space by an operator range of a Banach space with basis. Trans. Amer. Math. Soc., 358(4):1421-1434 (electronic), 2006.

[14] Su Gao. Unitary group actions and Hilbertian Polish metric spaces. In Logic and its applications, volume 380 of Contemp. Math., pages 53-72. Amer. Math. Soc., Providence, RI, 2005.

[15] Su Gao and Vladimir Pestov. On a universality property of some abelian Polish groups. Fund. Math., 179(1):1-15, 2003.

[16] G. Godefroy and N. J. Kalton. Lipschitz-free Banach spaces. Studia Math., 159(1):121-141, 2003.

[17] N. J. Kalton. Spaces of Lipschitz and Hölder functions and their applications. Collect. Math., $55(2): 171-217,2004$.

[18] N. J. Kalton and S. J. Montgomery-Smith. Set-functions and factorization. Arch. Math. (Basel), 61(2):183-200, 1993.

[19] Michael G. Megrelishvili. Private, e-mail communication.

[20] Michael G. Megrelishvili. Reflexively but not unitarily representable topological groups. Topology Proc., 25(Summer):615-625 (2002), 2000.

[21] Michael G. Megrelishvili. Topological transformation groups: selected topics. In Elliott Pearl, editor, Open problems in topology, pages 423-439. 2007.

[22] Vladimir Pestov. Free abelian topological groups and the Pontryagin-van Kampen duality. Bull. Austral. Math. Soc., 52(2):297-311, 1995.

[23] Vladimir Pestov. Amenable representations and dynamics of the unit sphere in an infinitedimensional Hilbert space. Geom. Funct. Anal., 10(5):1171-1201, 2000.

[24] Vladimir Pestov. Dynamics of infinite-dimensional groups and Ramsey-type phenomena. Instituto Nacional de Matemática Pura e Aplicada (IMPA), Rio de Janeiro, 2005.

[25] Vladimir Pestov. Forty annotated questions about large topological groups. In Elliott Pearl, editor, Open problems in topology, pages 439-451. Elsevier, 2007.

[26] Daniel J. Randtke. A simple example of a universal Schwartz space. Proc. Amer. Math. Soc., $37: 185-188,1973$.

[27] I. J. Schoenberg. Metric spaces and positive definite functions. Trans. Amer. Math. Soc., 44(3):522-536, 1938.

[28] Alexander I. Shtern. Compact semitopological semigroups and reflexive representability of topological groups. Russian J. Math. Phys., 2(1):131-132, 1994.

[29] M. G. Tkachenko. On the completeness of free abelian topological groups. Dokl. Akad. Nauk SSSR, 269(2):299-303, 1983.

[30] V. V. Uspenskiı. Free topological groups of metrizable spaces. Izv. Akad. Nauk SSSR Ser. Mat., 54(6):1295-1319, 1990.

[31] V. V. Uspenskiı̌. On the topology of a free locally convex space. Dokl. Akad. Nauk SSSR, 270(6):1334-1337, 1983.

[32] Vladimir Uspenskil. e-mail to the author, December 2005.

[33] Vladimir Uspenskii. Unitary representability of free abelian topological groups. Appl. Gen. Topology, to appear.

[34] Nik Weaver. Lipschitz algebras. World Scientific Publishing Co. Inc., River Edge, NJ, 1999.

Departmento de Matemáticas, Universidad Jaume I, Campus Riu Sec, 12071, Castellón SPAIN

E-mail address: jgalindo@mat.uji.es 